Research Article

\title{
Ka-Band Lightweight High-Efficiency Wideband 3D Printed Reflector Antenna
}

\author{
Yu Zhai, Ding Xu, and Yan Zhang \\ School of Electronic and Information Engineering, Beihang University, Beijing, China \\ Correspondence should be addressed to Yu Zhai; zhaiyu@buaa.edu.cn
}

Received 29 September 2017; Revised 30 October 2017; Accepted 6 November 2017; Published 25 December 2017

Academic Editor: Yu Jian Cheng

Copyright @ 2017 Yu Zhai et al. This is an open access article distributed under the Creative Commons Attribution License, which permits unrestricted use, distribution, and reproduction in any medium, provided the original work is properly cited.

\begin{abstract}
This paper presents a lightweight, cost-efficient, wideband, and high-gain 3D printed parabolic reflector antenna in the Ka-band. A $10 \lambda$ reflector is printed with polylactic acid- (PLA-) based material that is a biodegradable type of plastic, preferred in $3 \mathrm{D}$ printing. The reflecting surface is made up of multiple stacked layers of copper tape, thick enough to function as a reflecting surface (which is found $4 \mathrm{~mm}$ ). A conical horn is used for the incident field. A center-fed method has been used to converge the energy in the broadside direction. The proposed antenna results measured a gain of $27.8 \mathrm{dBi}$, a side lobe level (SLL) of $-22 \mathrm{~dB}$, and a maximum of $61.2 \%$ aperture efficiency (at $30 \mathrm{GHz}$ ). A near-field analysis in terms of amplitude and phase has also been presented which authenticates the accurate spherical to planar wavefront transformation in the scattered field.
\end{abstract}

\section{Introduction}

For long-distance wireless communication, for example, radio astronomy, satellite, radar, and microwave links, high-gain antennas are proffered. Parabolic reflectors and antennas designed with planar array theory fulfill the demand and being used in these applications for decades. Due to the presence of feeding structure in the planar array antennas, losses are generated due to conductor, dielectric, and undesired radiations from the corner bends which degrades the antenna performance. On the other hand, parabolic reflector uses spatial-fed method that overcomes the problem of feeding network and exhibits higher aperture efficiency as well as wider bandwidth. The only disadvantage in the reflector is its minimum beam scanning ability but still parabolic reflectors are being used in many applications due to their numerous advantages.

Parabolic reflector works on the principle of geometrical optics (GO) and physical optics (PO). It consists of two main parts, the feed and a reflecting surface. The feed is placed away from the reflector at the focal point. In transmit mode, the incident spherical wave from the feed is converted to planar wave-front while in receiving mode this planar wavefront is converted back into spherical wave. In this way, the energy is converged at the focal point of the reflector. In [1], a high-gain corner reflector is designed, where it is mentioned that the effect of antenna gain is mainly dependent on the angle of the corner. In the first part of [2], a detailed principle of the center-fed parabolic reflector is described, while in the second part, several practical feeds are discussed for these parabolic antennas. A theoretical analysis of the radiating properties of high-gain and low cross-polarized centerfed paraboloid reflector and the hyperboloid lens is described in [3]. The radiating mechanism of an off-axis feed reflector antenna with the help of computing various components of the field, aperture radiations, and direct feed radiation is discussed in [4]. In center-fed reflectors, due to the physical presence of the feed at the focal point, the reflected waves are blocked which degrades the antenna performance. To solve this problem, few alternative feeding methods are described in the literature such as offset feeding [5] and Cassegrain reflector [6] where the feed is behind the main reflecting surface.

In [7], the performance of reflector antenna is analyzed due to variation of the focal point. Several other reflector antennas are described in [8-12]. To make the coverage possible at multiple locations using a single reflector, multifeed and multibeam method is used, where a cluster of feeds 
are used to excite reflector surface currents, so for this purpose, a study on optimizing the performance of the feed is presented in [10]. The phase center of the feed cluster is aligned in a circular arc, passing through the phase center of the central feed at the focal point. It is important to mention here that when the feed phase center does not coincide with the focal point of the reflector, phase errors are generated that reduces the antenna performance. So, due to different edge tapering at the reflector, as well as the misalignment of the feed phase centers with the focal point, feed performance optimization is important.

It has been observed in the literature that the main disadvantage of parabolic reflector technology is its minimum beam scanning ability. To overcome this problem, several designs exist in literature in the recent years, which has resulted in $\pm 50^{\circ}$ beam scanning off the broadside [11-14].

In the recent years, 3D printing technology is exhibiting great potential in terms of lightweight and cost efficiency, when used in various applications. In [15-17], several 3D printed antennas are designed and fabricated operating at millimetre wave to terahertz frequency bands, using two different printing technologies: binder jetting/sintering on $316 \mathrm{~L}$ stainless steel and the selective laser melting (SLM) on $\mathrm{Cu}-15 \mathrm{Sn}$. In $[18,19]$, different microwave devices and guiding structure are designed using 3D printing technology. In [20], an offset-fed dual band 3D printed circularly polarized stepped reflector has been designed and measured. The antenna size is $400 \mathrm{~mm} \times 400 \mathrm{~mm}$, with a maximum thickness of $16.4 \mathrm{~mm}$. The antenna is designed with 4 cofocal paraboloids with focal length varying in $15 \mathrm{~mm}$, making a path difference of 2 to 3 wavelengths from $20 \mathrm{GHz}$ to $30 \mathrm{GHz}$. It is mentioned that the stepped reflector results in broader antenna bandwidth, while, due to the 4 planar steps in the stepped reflector, the reflector antenna resembles more with reflectarray than the conventional parabolic reflector. The total antenna weight is $0.9 \mathrm{~kg}$.

It is observed that although a $3 \mathrm{D}$ printed dual-band stepped reflector has already been designed with planar reflecting surfaces, designing an efficient and lightweight parabolic reflector with $3 \mathrm{D}$ printing still remains a challenge due to its curved surface. As we know, at the higher frequency bands, the surface roughness in the parabolic reflector creates phase errors that degrade the antenna performance, if parabolic surface is not accurately fabricated. In this paper, a new high-gain, lightweight, and cost-efficient 3D printed parabolic reflector antenna is proposed in the Ka-band. The reflecting surface is made of stacked layers of copper tape with a conductor thickness of $4 \mathrm{~mm}$. The proposed antenna results in wider matching bandwidth, gain bandwidth, and higher aperture efficiency.

\section{Conventional and Proposed 3D Printed Parabolic Reflector}

Parabolic reflector consists of two main parts: the feed and a reflecting surface, as shown in Figure 1. The feed is placed at the focal point coinciding the feed phase center. It generates spherical waves that travel towards the reflecting surface. The incident waves are reflected back towards the

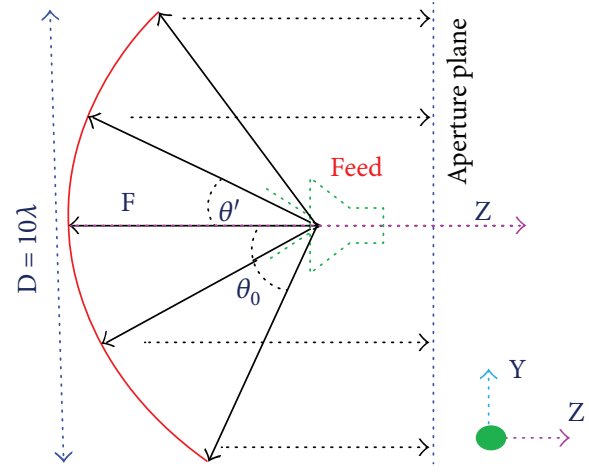

FIGURE 1: Basic principle of the parabolic reflector.

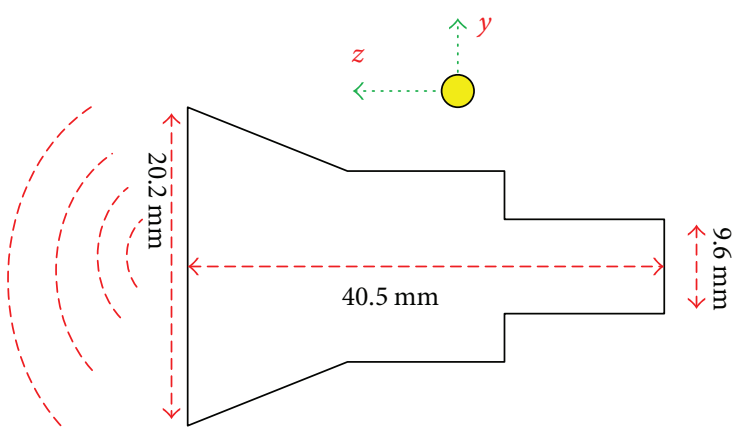

Figure 2: A Ka-band conical horn model used as a feed in the proposed 3D printed reflector antenna.

aperture plane converting the spherical wavefront to planar wavefront, as shown in Figure 1.

2.1. Linearly Polarized Feed. A linearly polarized conical horn is designed and fabricated to be used as a feed. The feed model is shown in Figure 2. The feed performance is optimized in terms of having wider matching bandwidth, minimum phase center variation versus frequency, and symmetrical radiation patterns in both $\mathrm{E}$ - and $\mathrm{H}$-planes. The symmetrical radiation pattern helps in resulting lower cross polarization from the reflector antenna. The feed radiation pattern is symmetrical in both planes, until $-20 \mathrm{~dB}$ from the peak. The simulated performance of the feed is shown in Figures 3, 4, 5, and 6. An edge tapering of $-10 \mathrm{~dB}$ is considered that results in $F / D=0.38$. The half-subtended angle is calculated using (1) and found $55^{\circ}$. To analyze the expected aperture efficiency from this particular feed, the feed pattern is assumed as a cosine distribution, as shown in (2). The exponent " $n$ " is found 4 , which fits well with the radiation pattern. The aperture efficiency is calculated, which is obtained by the product of multiple efficiencies, as shown in (3), where $\varepsilon_{\mathrm{s}}$ is spillover, $\varepsilon_{\mathrm{t}}$ is tapering, $\varepsilon_{\mathrm{p}}$ is phase, $\varepsilon_{\mathrm{x}}$ is polarization, $\varepsilon_{r}$ is the random phase error due to reflector surface, and $\varepsilon_{\mathrm{b}}$ is the feed blockage efficiency. It has been observed that the spillover and tapering efficiencies contribute the most in resulting aperture efficiency, while all other efficiencies are considered as 1 . The spillover and tapering efficiency is calculated using (4) and (5). The 


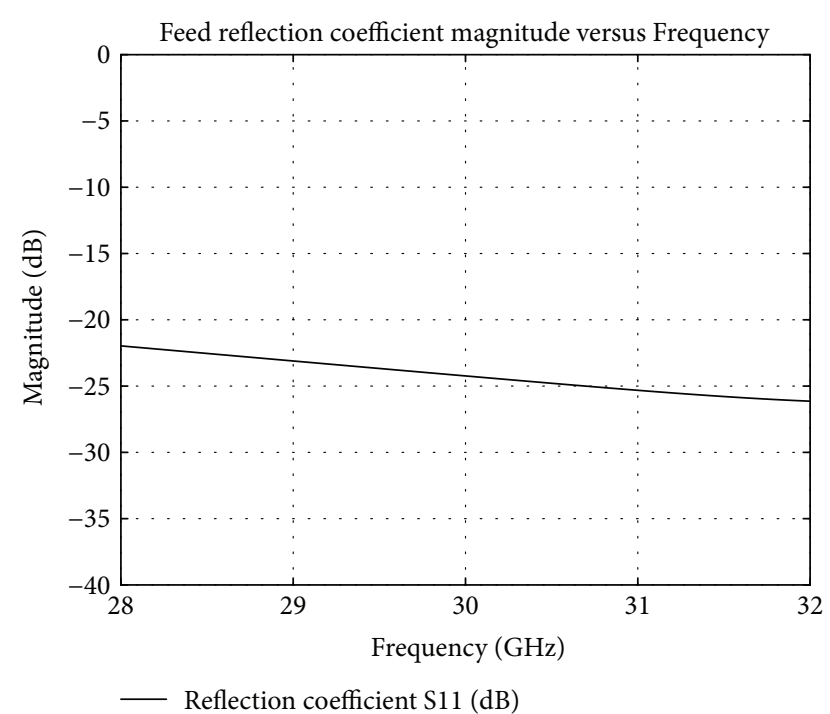

FIGURE 3: Simulated reflection coefficient magnitude versus frequency of the feed.

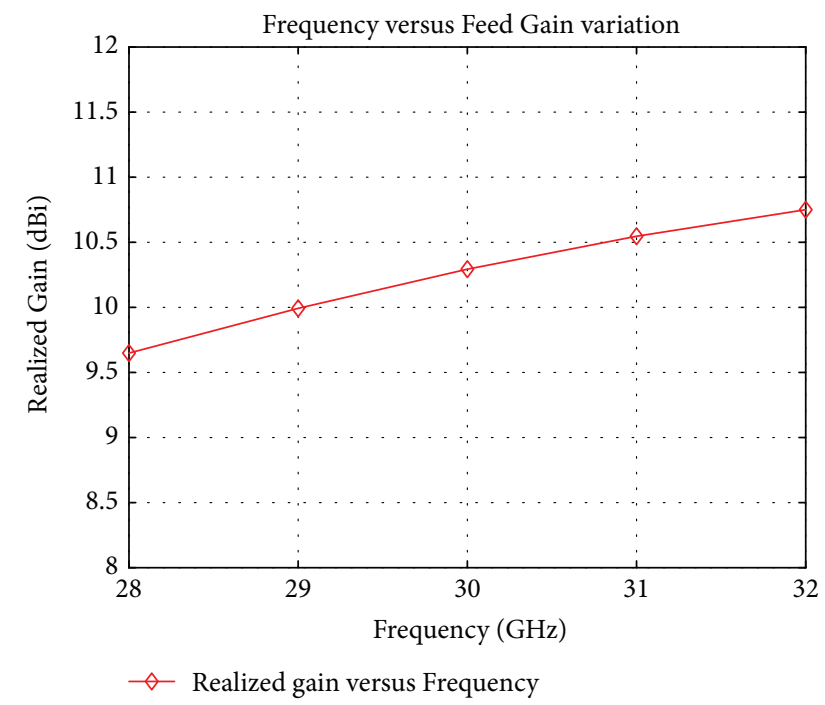

FIgURE 4: Simulated realized gain versus frequency of the feed.

highest possible aperture efficiency from this feed is expected 69\%, as shown in Figure 7.

$$
\begin{aligned}
\theta_{0} & =\tan ^{-1}\left(\frac{D / 2}{F}\right), \\
G_{\mathrm{f}}\left(\theta^{\prime}\right) & =\cos ^{n}\left(\theta^{\prime}\right), \\
\varepsilon_{\mathrm{ap}} & =\varepsilon_{\mathrm{s}} \varepsilon_{\mathrm{t}} \varepsilon_{\mathrm{p}} \varepsilon_{\mathrm{x}} \varepsilon_{\mathrm{r}} \varepsilon_{\mathrm{b}}, \\
\varepsilon_{\mathrm{s}} & =\frac{\int_{0}^{\theta_{0}} G_{f}\left(\theta^{\prime}\right) \sin \theta^{\prime} d \theta^{\prime}}{\int_{0}^{\pi} G_{f}\left(\theta^{\prime}\right) \sin \theta^{\prime} d \theta^{\prime}},
\end{aligned}
$$

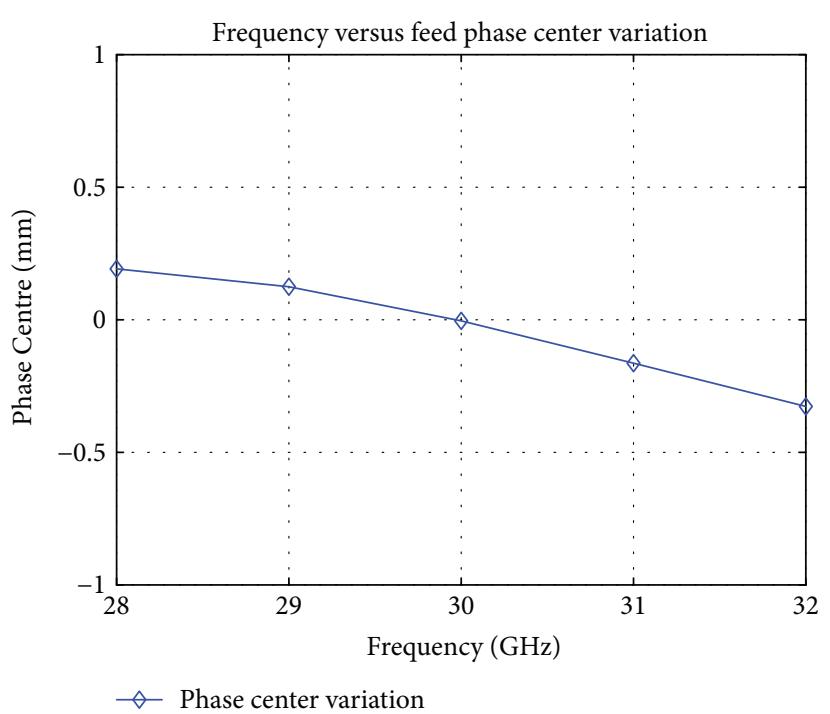

FIGURE 5: Simulated feed phase center versus frequency.

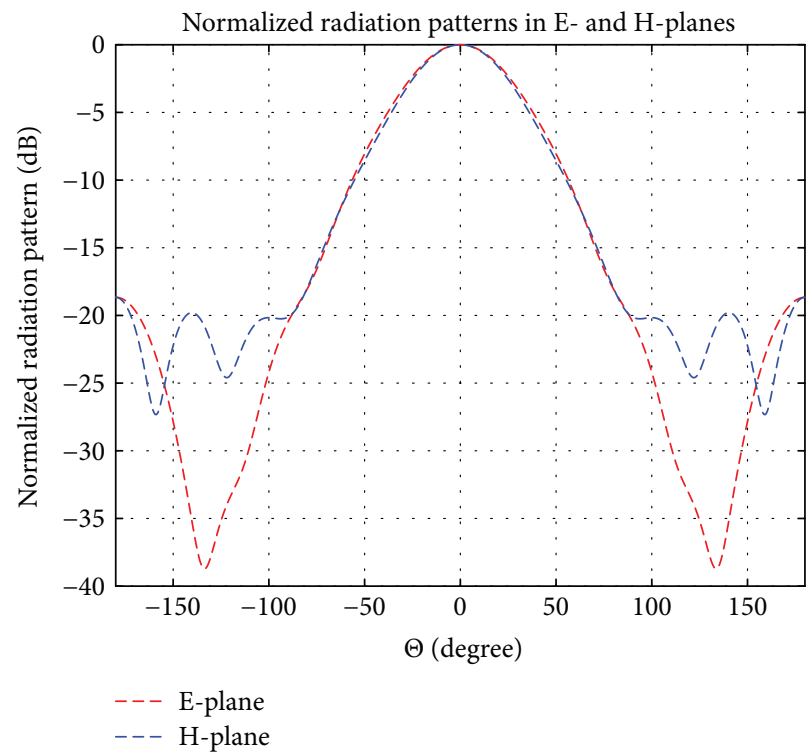

FIgURE 6: Simulated radiation pattern of the feed in E-plane and $\mathrm{H}$-plane at $30 \mathrm{GHz}$.

$$
\varepsilon_{\mathrm{t}}=2 \cot ^{2}\left(\frac{\theta_{0}}{2}\right) \frac{\left|\int_{0}^{\theta_{0}} G_{\mathrm{f}}\left(\theta^{\prime}\right) \tan \left(\theta^{\prime} / 2\right) d \theta^{\prime}\right|^{2}}{\int_{0}^{\theta_{0}} G_{\mathrm{f}}\left(\theta^{\prime}\right) \sin \theta^{\prime} d \theta^{\prime}} .
$$

2.2. 3D Printed Reflector Model. A diameter of $10 \lambda$ parabolic reflector is designed using PLA-based plastic material, where hexagonal-shaped filling is used, which makes the design lightweight but still mechanically very strong to handle, as shown in Figure 8. The total weight of the proposed antenna is $0.2 \mathrm{~kg}$. The reflecting surface is made up of copper tape. During the measurements, it has been observed that using a single layer of copper tape did not act as a completely reflecting surface but a partially transmitting surface due to skin depth of the waves at this frequency 


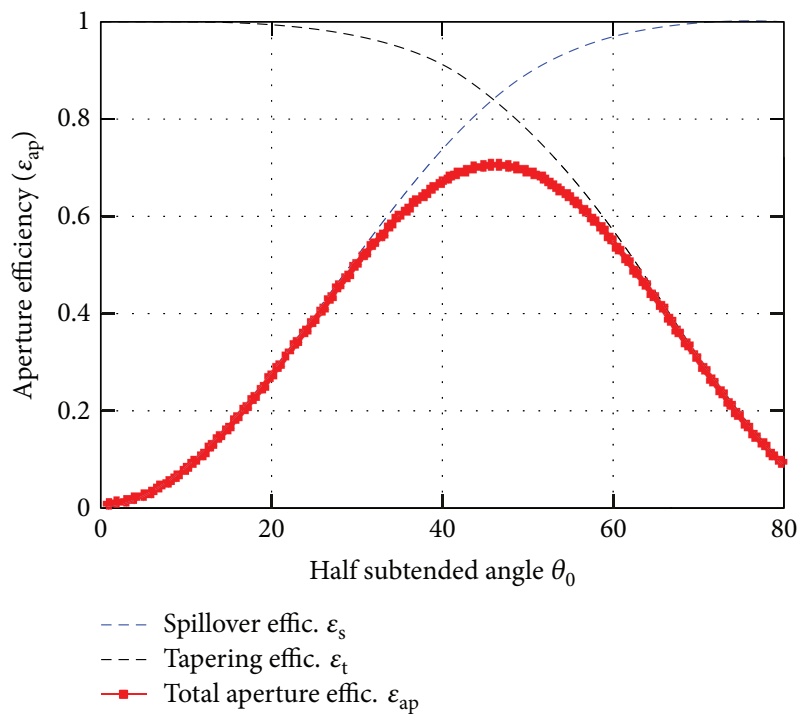

FIGURE 7: Simulated aperture efficiency versus half subtended angle of the reflector.

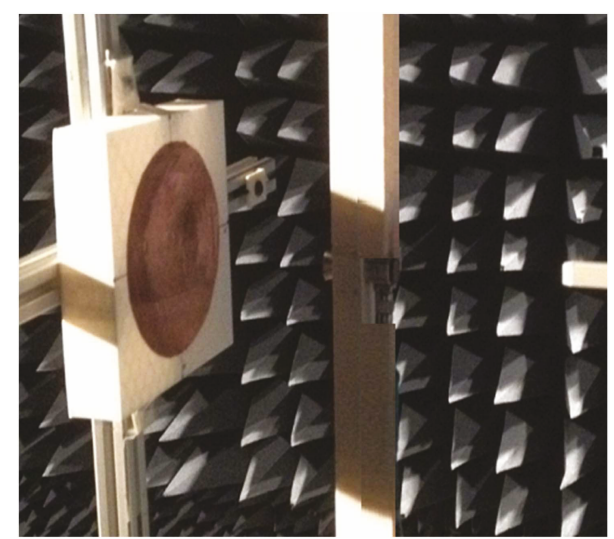

Figure 8: A picture of the fabricated 3D printed reflector antenna.

band. In the measurement, a higher signal penetration is observed than the reflection. To solve this problem, multiple rounds of measurements are taken by making the reflecting surface thicker and thicker. When the reflecting surface thickness went over $3 \mathrm{~mm}$, the measurements started making agreement with the simulations. So, for the safe end, the reflecting surface is made up of thickness of $4 \mathrm{~mm}$ for taking the final measurements, and the results are presented in this paper.

2.3. Simulated and Measured Results. Near-field measurements are taken which provide a maximum far-field scan angle of $\pm 30^{\circ}$. Through near-field, aperture fields are measured which are further used to calculate far-field radiation patterns. As the scan angle is bounded by $\pm 30^{\circ}$, so field varying around the main beam, and in the first couple of side lobes, is captured but the prediction about the far away side lobes and back lobes cannot be predicted with this scan angle. The selection of this particular scan angle is limited to

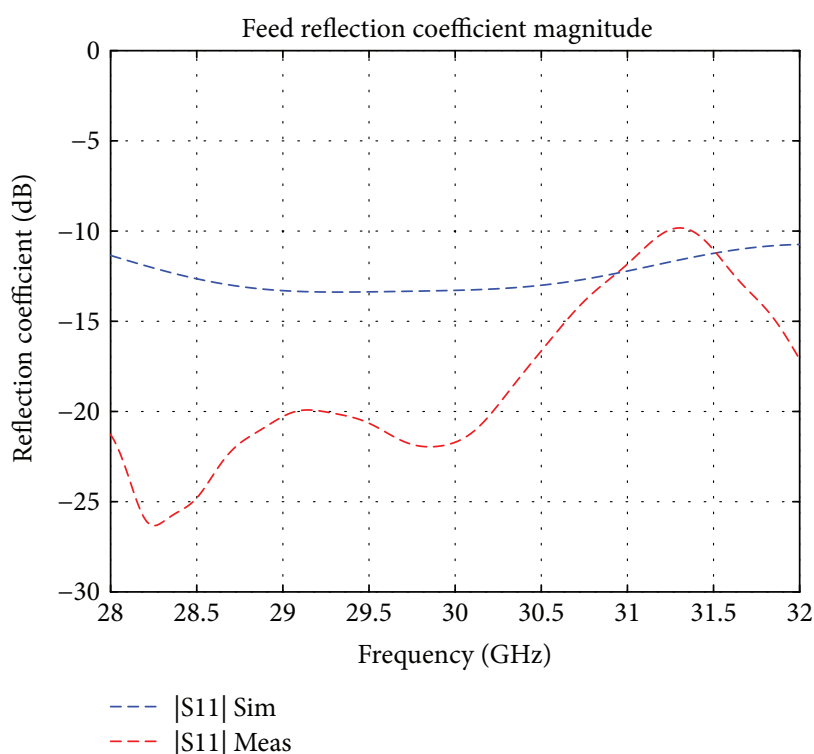

FIGURE 9: Simulated and measured reflection coefficient magnitude versus frequency from the proposed reflector.

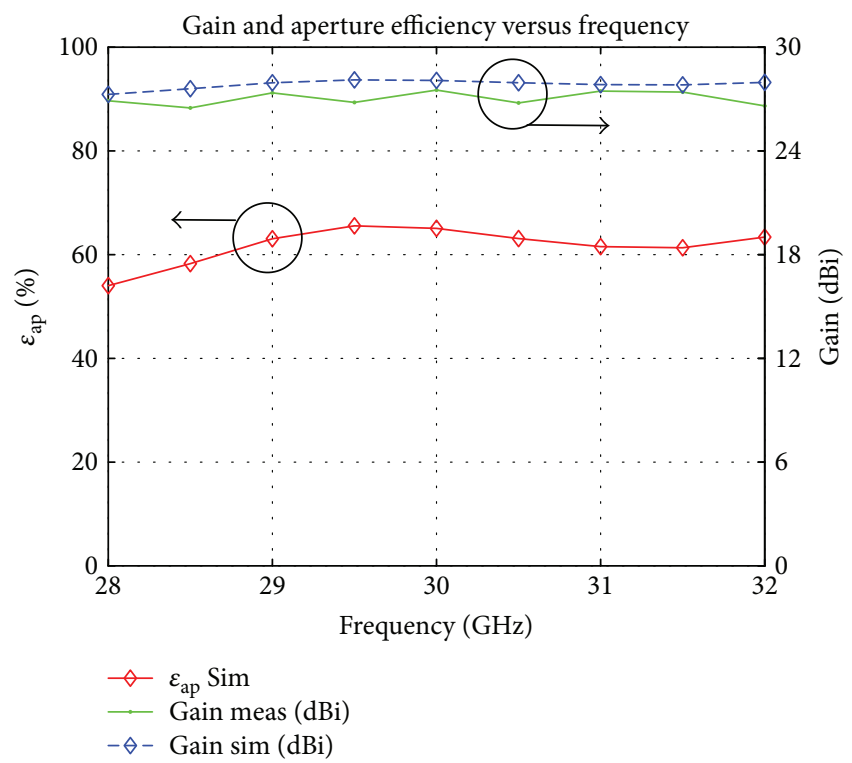

FIgURE 10: Simulated and measured gain versus frequency from the proposed reflector.

the available measurement resources in our university. The transformation from near-field to far-field is achieved automatically by the used Agilent network analyzer, using Fourier transformation. The measured antenna performance is compared with simulation, in terms of reflection coefficient shown in Figure 9, realized gain and aperture efficiency shown in Figure 10, the E-plane radiation patterns shown in Figure 11, and the H-plane radiation patterns shown in Figure 12, which result in reasonable agreement. The proposed antenna results in measured matching bandwidth of $13.34 \%$ (while the threshold of $-10 \mathrm{~dB}$ is considered), the highest measured gain of $27.8 \mathrm{dBi}$, almost a constant gain in 


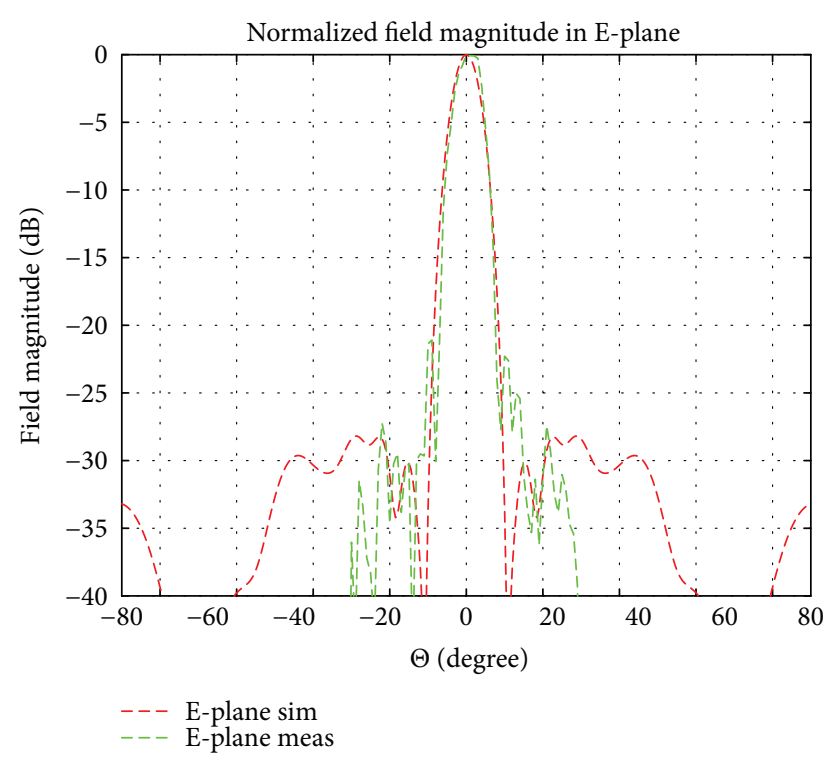

Figure 11: Simulated and measured E-plane radiation patterns from the proposed $3 \mathrm{D}$ printed reflector antenna at $30 \mathrm{GHz}$.

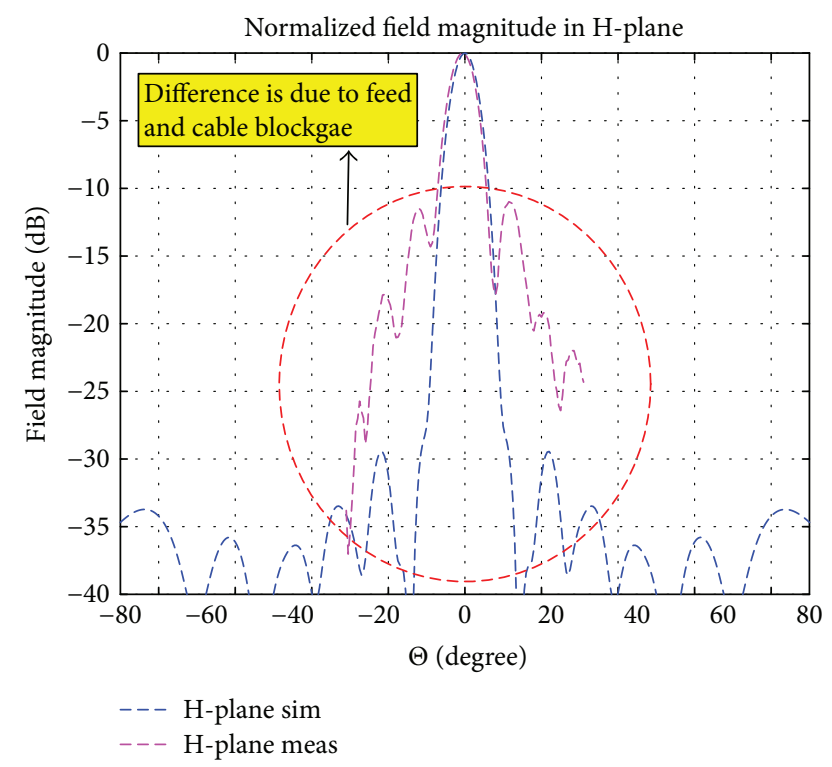

FIGURE 12: Simulated and measured H-plane radiation patterns from the proposed $3 \mathrm{D}$ printed reflector antenna at $30 \mathrm{GHz}$.

the whole frequency band (varying within $0.5 \mathrm{dBi}$ ), SLL of $-22 \mathrm{~dB}$ in the E-plane, and the highest aperture efficiency of $61.2 \%$ (at $30 \mathrm{GHz}$ ). It is also important to mention that in the above feed analysis section, we have observed that the expected highest aperture efficiency is $69 \%$ while the measured is $61.2 \%$. This difference between simulation and measurements is because of either feed and cable blockage or focal point and feed phase center misalignment or due to the edge diffraction or their accumulation. The proposed 3D printed reflector antenna exhibits high gain, wider matching and gain bandwidth, and low cost. The proposed
TABLE 1: The proposed 3D printed reflector antenna measured performance at $30 \mathrm{GHz}$.

\begin{tabular}{cccccc}
\hline Measured & $\begin{array}{c}\text { Impedance } \\
\mathrm{BW}(\%)\end{array}$ & $\begin{array}{c}\text { Max gain } \\
(\mathrm{dBi})\end{array}$ & $\begin{array}{c}1 \mathrm{~dB} \text { gain } \\
\mathrm{BW}(\%)\end{array}$ & $\begin{array}{c}\text { SLL } \\
(\mathrm{dB})\end{array}$ & $\begin{array}{c}\varepsilon_{\text {ap }} \\
(\%)\end{array}$ \\
\hline Proposed & 13.4 & 27.8 & 13.4 & -22 & 61.2 \\
\hline
\end{tabular}

antenna is broadband, lightweight, easier in fabrication, and cost-efficient. The proposed antenna performance is summarized in Table 1. The performance of the proposed antenna is also compared with [20] and summarized in Table 2.

2.4. Near-Field Analysis. One basic principle of reflector antenna is to convert the incident spherical wavefront from the feed to planar wavefront in the scattered field or vice versa. So, to authenticate this conversion, near-field analysis is performed in terms of incident, total, and scattered field. The incident electric field and total field distributions are not shown here for brevity but only the scattered field results are presented. As the incident field is Y-polarized, so the copolar scattered field amplitude and phase distribution will authenticate the accurate wavefront transformation. The copolar scattered field amplitude and phase distribution taken at a plane $z=45 \mathrm{~mm}$ (behind the feed not to include the feed errors) are shown in Figures 13 and 14, those not only verify the amplitude tapering from center to the edge of reflector but also the copolar scattered field phase distribution, which is constant. These results exhibit that the incident spherical wavefront is converted to planar wavefront.

\section{Conclusion}

This paper has presented a lightweight, cost-efficient, and high-gain $3 \mathrm{D}$ printed parabolic reflector antenna in the Ka-band. A reflector diameter of $10 \lambda$ has been designed and fabricated using polylactic acid- (PLA-) based plastic material. The reflecting surface is made up of multiple stacked layers of copper tape, thick enough to function as a reflecting surface (which is found $4 \mathrm{~mm}$ ). During the study, it has been observed that for a 3D printed reflector design, a thick copper layer is more suitable for obtaining accurate antenna performance than a thin copper layer, due to the skin depth phenomenon at this frequency band. A conical horn has been used for the incident field, which further generates the surface currents at the reflector. A center-fed method has been used to converge the energy in the broadside direction and to achieve minimum cross polarization. The proposed antenna has resulted in a measured gain of $27.8 \mathrm{dBi}$, a side lobe level (SLL) of $-22 \mathrm{~dB}$, and the highest aperture efficiency of $61.2 \%$ (at $30 \mathrm{GHz}$ ). A near-field analysis in terms of amplitude and phase has also been presented that has authenticated the incident spherical wavefront converted to planar wavefront in the scattered field. 
TABLE 2: The performance comparison of the proposed 3D printed reflector antenna with the previous published in [20].

\begin{tabular}{|c|c|c|c|c|c|c|c|}
\hline Meas & Freq band $(\mathrm{GHz})$ & Size $(\mathrm{mm})$ & Weight (Kg) & Shape & Pol & SLL (dB) & Feed method \\
\hline Prop & $\mathrm{Ka}(30)$ & $100 \mathrm{~mm}^{2}$ & 0.2 & Parabolic & LP & -22 & Center fed \\
\hline$[20]$ & $K-K a(20 / 30)$ & $400 \mathrm{~mm}^{2}$ & 0.9 & Stepped planar & $\mathrm{CP}$ & -20 & Offset fed \\
\hline
\end{tabular}

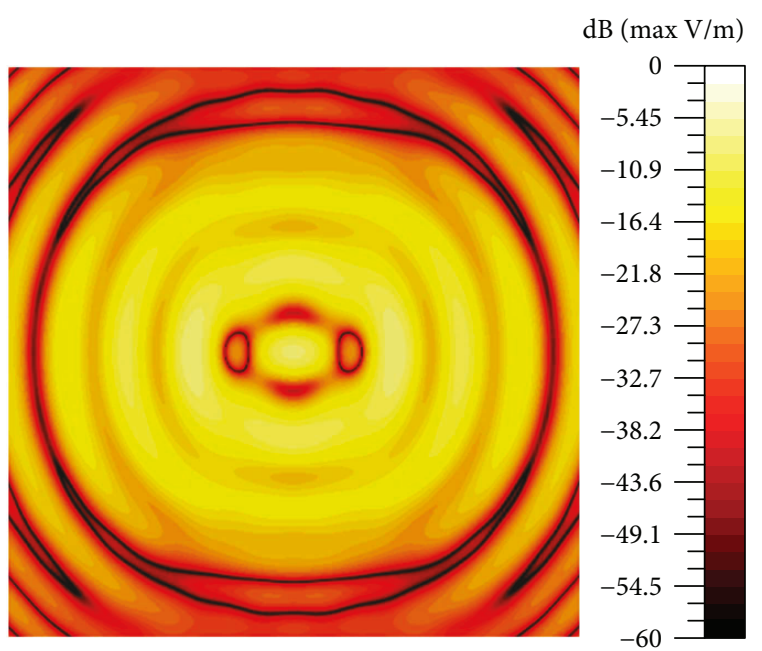

Figure 13: Simulated scattered field copolar amplitude distribution plot at $z=45 \mathrm{~mm}$ plane (behind the feed).

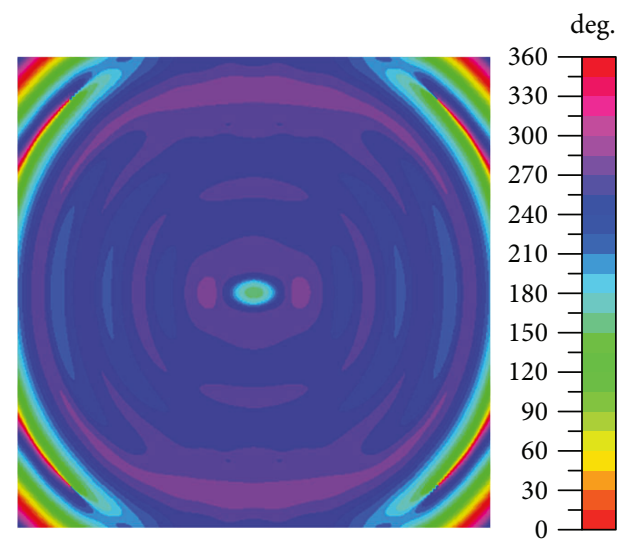

FIGURE 14: Simulated scattered field copolar phase distribution plot at $z=45 \mathrm{~mm}$ plane (behind the feed).

\section{Conflicts of Interest}

The authors declare that they have no conflicts of interest.

\section{References}

[1] J. D. Kraus, “The corner-reflector antenna," Proceedings of the IRE, vol. 28, no. 11, pp. 513-519, 1940.

[2] C. C. Cutler, "Parabolic-antenna design for microwaves," Proceedings of the IRE, vol. 35, no. 11, pp. 1284-1294, 1947.

[3] E. Jones, "Paraboloid reflector and hyperboloid lens antennas," Transactions of the IRE Professional Group on Antennas and Propagation, vol. 2, no. 3, pp. 119-127, 1954.
[4] S. Sandler, "Paraboloidal reflector patterns for off-axis feed," IRE Transactions on Antennas and Propagation, vol. 8, no. 4, pp. 368-379, 1960

[5] W. Strutzman and M. Terada, "Design of offset-parabolicreflector antennas for low cross-pol and low sidelobes," IEEE Antennas and Propagation Magazine, vol. 35, no. 6, pp. 4649, 1993.

[6] E. Wilkinson and A. Appelbaum, "Cassegrain systems," IRE Transactions on Antennas and Propagation, vol. 9, no. 1, pp. 119-120, 1961.

[7] H. Ling, S.-W. Lee, P. Lam, and W. Rusch, "Focal shifts in parabolic reflectors," IEEE Transactions on Antennas and Propagation, vol. 33, no. 7, pp. 744-748, 1985.

[8] D. C. Chang, C. C. Yang, and S. Y. Yang, "Dual-reflector system with a spherical main reflector and shaped subreflector for compact range," IEE Proceedings - Microwaves, Antennas and Propagation, vol. 144, no. 2, pp. 97-102, 1997.

[9] M. A. B. Terada and W. L. Stutzman, "Computer-aided design of reflector antennas: the Green Bank Radio Telescope," IEEE Transactions on Microwave Theory and Techniques, vol. 46, no. 3, pp. 250-253, 1998.

[10] J. Kuecken, "Feed optimization in multi-feed antennas," in WESCON/57 Conference Record, pp. 164-179, San Francisco, CA, USA, August 1957.

[11] K. Bahadori and Y. Rahmat-Samii, "An array-compensated spherical reflector antenna for a very large number of scanned beams," IEEE Transactions on Antennas and Propagation, vol. 53, no. 11, pp. 3547-3555, 2005.

[12] Y. J. Cheng, W. Hong, and K. Wu, "Millimeter-wave substrate integrated waveguide multibeam antenna based on the parabolic reflector principle," IEEE Transactions on Antennas and Propagation, vol. 56, no. 9, pp. 3055-3058, 2008.

[13] R. Yang, W. Tang, and Y. Hao, "Wideband beam-steerable flat reflectors via transformation optics," IEEE Antennas and Wireless Propagation Letters, vol. 10, pp. 1290-1294, 2011.

[14] A. Hosseini, S. Kabiri, and F. De Flaviis, " $V$-band high-gain printed quasi-parabolic reflector antenna with beam-steering," IEEE Transactions on Antennas and Propagation, vol. 65, no. 4, pp. 1589-1598, 2017.

[15] B. Zhang, Z. Zhan, Y. Cao et al., "Metallic 3-D printed antennas for millimeter- and submillimeter wave applications," IEEE Transactions on Terahertz Science and Technology, vol. 6, no. 4, pp. 592-600, 2016.

[16] B. Zhang and H. Zirath, "Metallic 3-D printed rectangular waveguides for millimeter-wave applications," IEEE Transactions on Components, Packaging and Manufacturing Technology, vol. 6, no. 5, pp. 796-804, 2016.

[17] B. Zhang, Y. X. Guo, H. Zirath, and Y. P. Zhang, "Investigation on 3-D-printing technologies for millimeter- wave and terahertz applications," Proceedings of the IEEE, vol. 105, no. 4, pp. 723-736, 2017.

[18] A. A. Muller, E. Sanabria-Codesal, A. Moldoveanu, V. Asavei, and S. Lucyszyn, "Extended capabilities of the 3-D smith chart 
with group delay and resonator quality factor," IEEE Transactions on Microwave Theory and Techniques, vol. 65, no. 1, pp. 10-19, 2017.

[19] M. D'Auria, W. J. Otter, J. Hazell et al., “3-D printed metal-pipe rectangular waveguides," IEEE Transactions on Components, Packaging and Manufacturing Technology, vol. 5, no. 9, pp. 1339-1349, 2015.

[20] L. G. Menendez, O. S. Kim, F. Persson, M. Nielsen, and O. Breinbjerg, "3D printed $20 / 30-\mathrm{GHz}$ dual-band offset stepped-reflector antenna," in 2015 9th European Conference on Antennas and Propagation (EuCAP), pp. 1-2, Lisbon, Portugal, April 2015. 


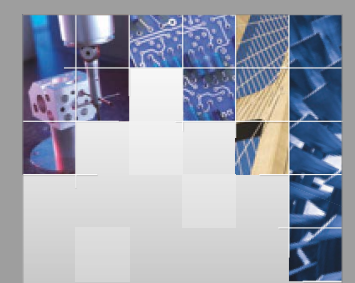

\section{Enfincering}
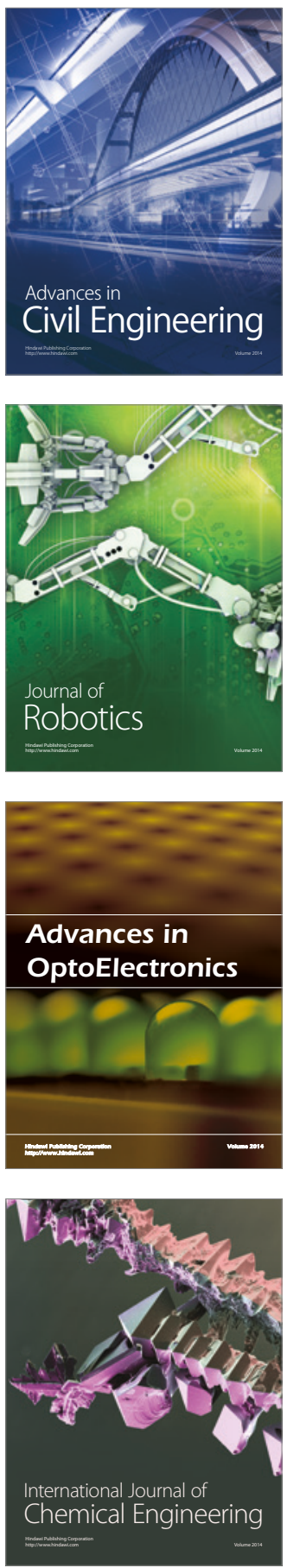

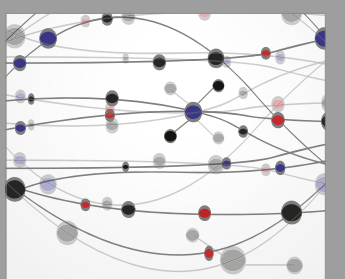

The Scientific World Journal

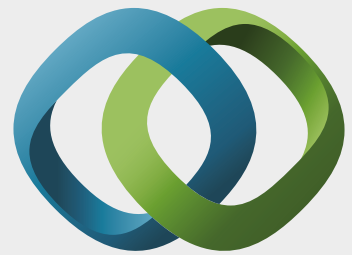

\section{Hindawi}

Submit your manuscripts at

https://www.hindawi.com
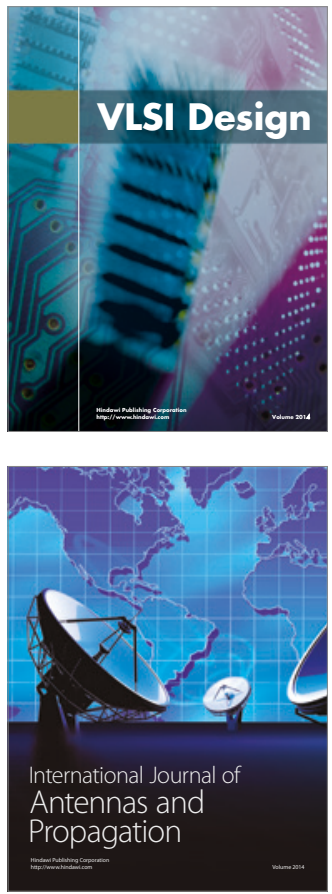

\section{Rotating}

Machinery
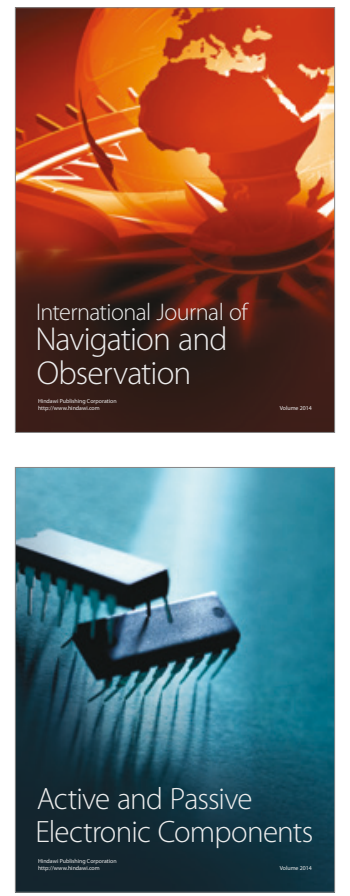
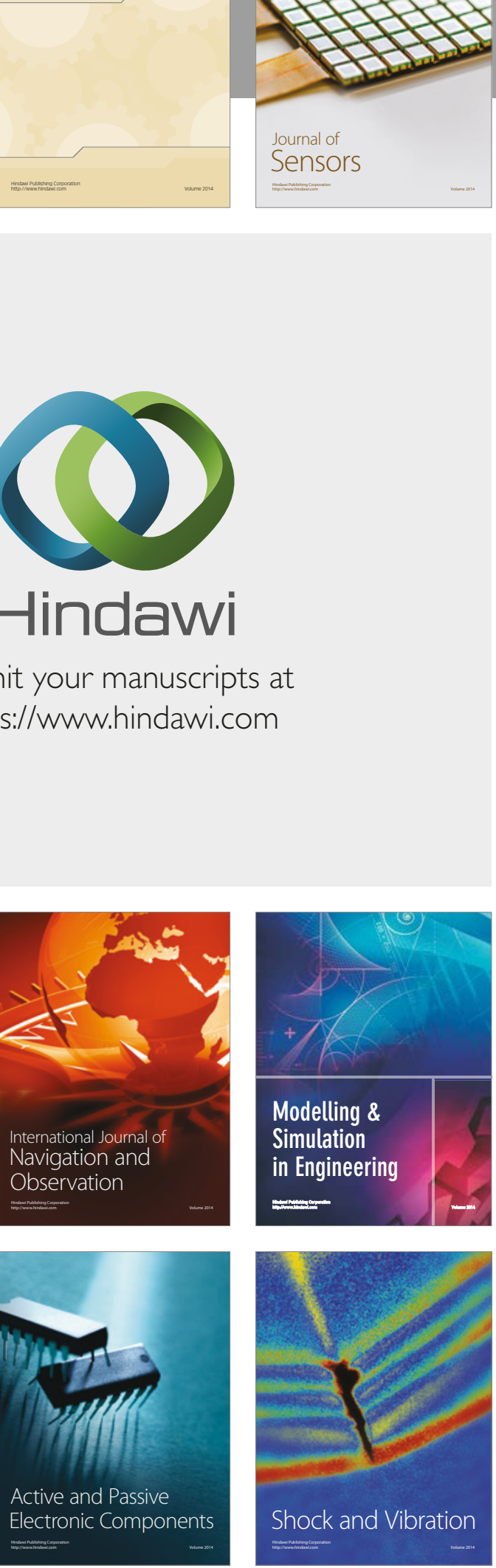
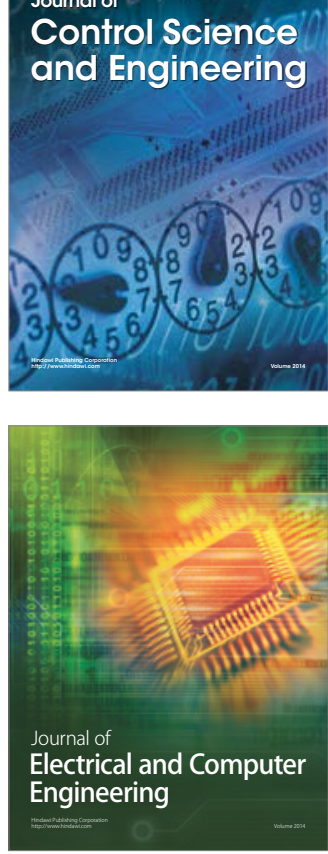

Distributed

Journal of

Control Science

and Engineering
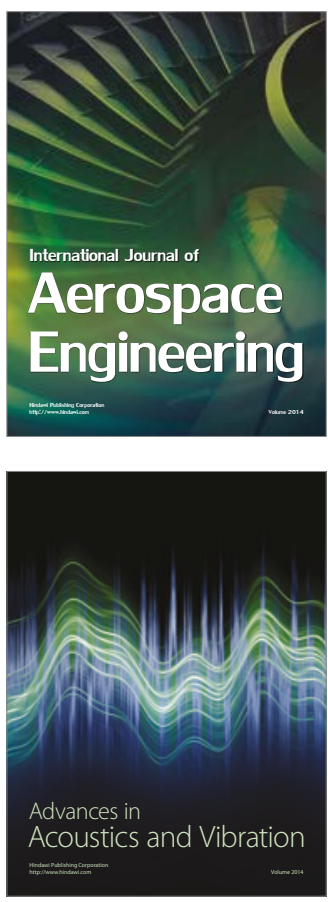

Sensor Networks 